\title{
Use of Pneumococcal Disease Epidemiology to Set Policy and Prevent Disease during 20 Years of the Emerging Infections Program
}

\author{
Matthew R. Moore, Cynthia G. Whitney
}

Two decades ago, the Emerging Infections Program of the US Centers for Disease Control and Prevention implemented what seemed like a simple yet novel idea: a population- and laboratory-based surveillance system designed to identify and characterize invasive bacterial infections, including those caused by Streptococcus pneumoniae. This system, known as Active Bacterial Core surveillance, has since served as a flexible platform for following trends in invasive pneumococcal disease and studying vaccination as the most effective method for prevention. We report the contributions of Active Bacterial Core surveillance to every pneumococcal vaccine policy decision in the United States during the past 20 years.

Ctreptococcus pneumoniae, or pneumococcus, is the most common bacterial vaccine-preventable cause of death in the United States; globally pneumococcus is responsible for 476,000 deaths annually among children $<5$ years of age (1). Most of these deaths occur in developing countries. However, early efforts by the Emerging Infections Programs (EIPs) to track pneumococcus in the United States grew out of concerns regarding increasing antimicrobial resistance in the early 1990s. At that time, the Centers for Disease Control and Prevention noted an increase in drug-resistant strains reported through its passive, sentinel, hospital-based surveillance system (2) and determined that more intensive tracking of pneumococcal disease was needed.

Surveillance for invasive pneumococcal disease (IPD) began in 1995 as part of the EIP/Active Bacterial Core surveillance (ABCs) programs in California, Connecticut, Georgia, Maryland, Minnesota, Oregon, and Tennessee. IPD, defined for this program as isolation of pneumococcus from a normally sterile site, was chosen as the syndrome to be tracked because pneumococci identified from blood or cerebrospinal fluid are indicative of disease, whereas pneumococci from the respiratory tract

Author affiliation: Centers for Disease Control and Prevention, Atlanta, Georgia, USA

DOI: http://dx.doi.org/10.3201/eid2109.150395 might not be indicative, and because clinical practices associated with severe disease were unlikely to vary dramatically in different geographic areas. Audits of clinical laboratories, which can be performed during in-person visits or by electronic queries, aimed to ensure that all cases of IPD in EIP sites were ascertained. Extensive reviews of medical records enable investigators to ascertain underlying conditions, as well as discharge status. The population under surveillance in 1996 was >19 million, but sites were added in 1997, 2000, and 2004. Population growth within each site has increased the total population under surveillance to 31 million in 2014. ABCs have reported estimates of disease burden every year since 1998 (http://www. cdc.gov/abcs). A more detailed presentation of methods used in ABCs is provided by Langley et al. elsewhere in this issue (3).

Since the inception of ABCs, numerous publications have drawn heavily on primary analysis of ABCs pneumococcal data, and many others have incorporated secondary analyses of data published in peer-reviewed literature. Some of the most influential outputs have focused on basic descriptive epidemiology. For example, EIP/ABCs data on antimicrobial resistance among pneumococci causing IPD helped shape treatment policy for pneumonia and meningitis $(4,5)$. A seminal paper containing data collected during 1995-1998 highlighted the increased risk for disease among children $<2$ years of age and adults $\geq 65$ years of age, as well as substantial racial disparity (greater risk for black persons vs. white persons) in every age group (6). In addition, the analysis showed that $59 \%$ of disease among adults 18-64 years of age occurred in persons who had an indication for receiving 23-valent pneumococcal polysaccharide vaccine (PPV23). However, vaccine coverage was and remains unacceptably low. An estimated 48,000 cases (76\%) of IPD and 5,300 deaths (87\%) occurred annually among persons who were eligible for pneumococcal vaccines at that time. This analysis, which was conducted as pneumococcal conjugate vaccines were undergoing clinical trials, helped to highlight the need to include the conjugate vaccine in the US pediatric vaccine schedule and to improve use of PPV23 among adults. 


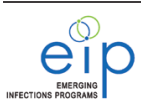

Having a solid surveillance infrastructure in place has provided major opportunities for EIPs to conduct special studies. One of the earliest with key policy implications was the Preventability Study, which was designed to evaluate the extent to which addition of proposed new Advisory Committee on Immunization Practices (ACIP) indications for PPV23 might increase the proportion of IPD preventable through better immunization coverage (7). In 2000, the age for universal influenza vaccination was reduced from 65 to 50 years of age $(8)$. This reduction raised the question of whether PPV23, which is frequently given to adults along with influenza vaccine, should also be administered to adults $\geq 50$ years of age. In the Preventability Study, EIP investigators interviewed 1,705 adults who had recovered from IPD to identify all providers from whom they had received care. The EIPs then determined which patients had already received PPV23 and, among those who had not, which patients had at $\geq 1$ ACIP indication for PPV23. Ultimately, the existing recommendations were proven to capture most adults with IPD, and the extant data were not sufficient to support reducing the age of universal vaccination with PPV23 (7).

Early EIP data served a major baseline for assessing the benefits of introduction of 7-valent pneumococcal conjugate vaccine (PCV7, Prevnar; Pfizer, Pearl River, NY, USA) in 2000. PCV7 was licensed on the basis of a randomized controlled trial in The Northern California Kaiser Permanente health care system, which demonstrated 97\% efficacy against PCV7-serotype IPD when administered on a schedule of dosing at 2, 4, 6, and 12-15 months of age (9). The ACIP recommended use of PCV7 on that schedule (10), and the American Academy of Pediatrics issued similar recommendations (11). In 2001, a shortage of PCV7 led ACIP to recommend suspension of the booster (fourth) dose for healthy children (12) and, in 2003, a second shortage led to suspension of the third and fourth doses for healthy children (13). Although these shortages were unfortunate, they provided the EIPs with an opportunity to evaluate reduced-dose schedules, something which would have been challenging in the context of a randomized controlled trial.
The EIPs conducted a case-control study of PCV7 effectiveness during 2001-2003 and ultimately enrolled 782 case-patients and 2,512 controls (14). Effectiveness of $\geq 1$ doses of PCV7 against PCV7-type IPD was 96\%, and estimates of serotype-specific effectiveness were strikingly similar to those from the Kaiser trial (Table). However, because of the shortages, EIPs were able to demonstrate that virtually any PCV7 schedule with $\geq 2$ doses in the first 6 months of life was 95\% effective in preventing PCV7type IPD. A schedule of 2 doses in the first 6 months, followed by a booster dose, was $98 \%$ effective. This 2 +1 schedule was subsequently adopted widely in many countries. EIP/ABCs surveillance documented a 94\% reduction in disease among children $<5$ years of age in the United States by 2003, in spite of the widespread shortages (Figure 1) (15).

Because the posterior nasopharynx had long been recognized as the reservoir for pneumococci, studies of asymptomatic colonization provided many insights into the dynamics of pneumococcal transmission. Multiple studies of the effects of pneumococcal conjugate vaccines on nasopharyngeal colonization demonstrated that vaccination prevents acquisition of vaccine-type pneumococci (16). This finding resulted in the hypothesis that widespread vaccination of children might reduce transmission to and ultimately, disease in adults. Herd protection conferred by PCV7 was far greater than predicted. Within the first 3 years of the PCV7 program in the United States, rates of PCV7-type IPD among adults began to decrease (17) and continued to decrease over subsequent years (Figure 2) (18). Whereas a primary driver of cost-effectiveness of PCV7 before introduction was the anticipated effect on otitis media visits among children, a key driver after introduction was the reduction in adult disease (19), something only identifiable through population-based surveillance. The cost per IPD episode averted without consideration of herd protection was $\$ 33,000$, and the cost per episode averted with herd protection decreased to $\$ 5,500$. This observation fundamentally changed the method for cost-effectiveness analyses of pneumococcal conjugate vaccines, not only in the United States $(20,21)$ but also

Table. Comparison of serotype-specific effectiveness of PCV7 (EIP/ABCs case-control study) (14) with that of NCKP trial (9) against invasive pneumococcal disease*

\begin{tabular}{|c|c|c|}
\hline \multirow[b]{2}{*}{ Serotype } & \multicolumn{2}{|c|}{ Vaccine effectiveness/efficacy, \% (95\% Cl) } \\
\hline & $\mathrm{CDC} / \mathrm{ABCs}$ & NCKP trial 2000 \\
\hline All PCV7 types & Healthy: 96 (93-98); underlying illness: 81 (57-92) & $94(80-98)$ \\
\hline 4 & $93(65-99)$ & NA \\
\hline $6 B$ & $94(77-98)$ & $86(-11$ to 100$)$ \\
\hline $9 \mathrm{~V}$ & $100(88-100)$ & $100(-142$ to 100$)$ \\
\hline 14 & $94(81-98)$ & $100(60-100)$ \\
\hline $18 \mathrm{C}$ & 97 (85-99) & $100(49-100)$ \\
\hline $19 F$ & 87 (65-95) & $85(32-98)$ \\
\hline $23 \mathrm{~F}$ & $98(80-100)$ & $100(15-100)$ \\
\hline
\end{tabular}

California Kaiser Permanente; CDC, Centers for Disease Control and Prevention; NA, not available. 


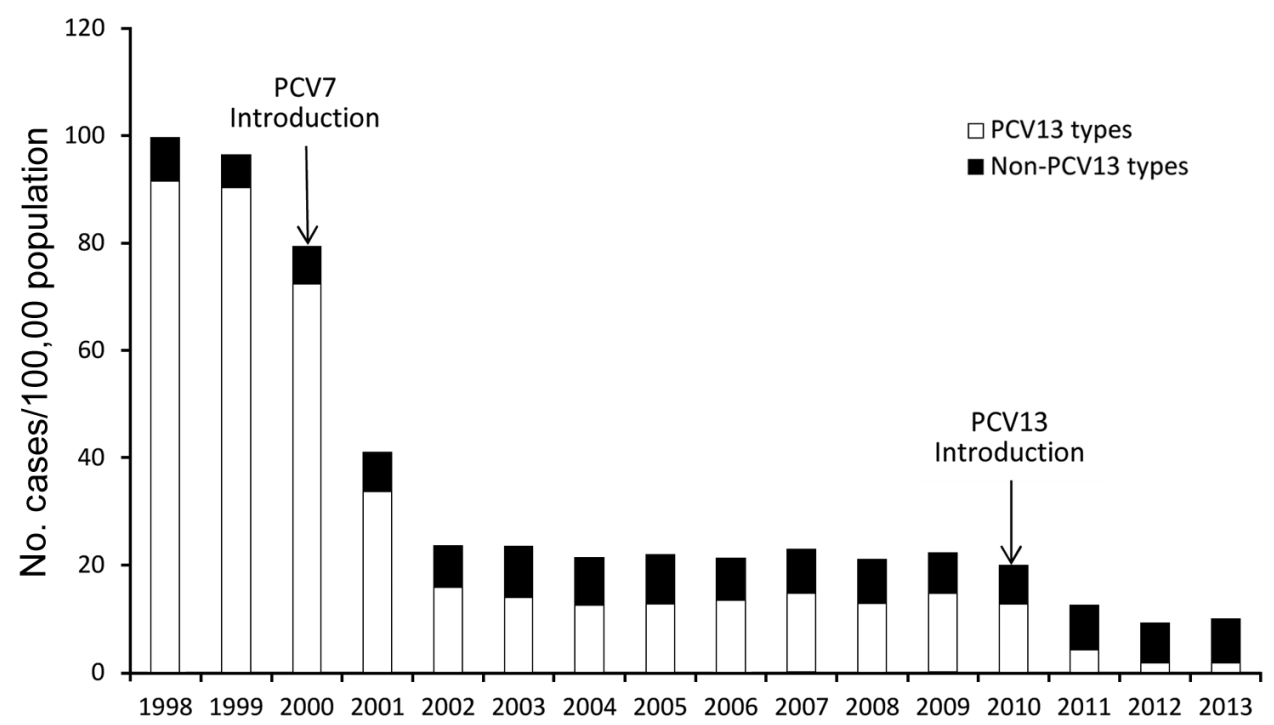

Figure 1. Incidence of invasive pneumococcal disease among children $<5$ years of age, caused by Streptococcus pneumoniae serotypes included in the 13-valent pneumococcal conjugate vaccine (PCV13) and by non-PCV13 serotype, Centers for Disease Control and Prevention Emerging Infections Program/Active Bacterial Core surveillance, 1998-2013.

in other countries $(22,23)$. A subsequent analysis, which incorporated the effect on pneumonia from non-EIP data sources, found PCV7 to be cost-saving (i.e., improved health outcomes at lower costs) (20).

During subsequent years, as shortages resolved, vaccine coverage increased and disease caused by PCV7 serotypes decreased, and EIPs detected an increase in rates of IPD caused by serotypes not included in PCV7. Even larger increases were described by other investigators in Alaska (24). A leading hypothesis was that these increases might represent serotype replacement, the process by which reductions in vaccine types open an ecologic niche for increases in nonvaccine serotypes in the nasopharynx, which ultimately lead to an increase in disease caused by nonvaccine serotypes. This phenomenon had been described in multiple randomized controlled trials of the effects of pneumococcal conjugate vaccines on nasopharyngeal colonization (16) but had never been described in the setting of invasive disease. Periodic increases and decreases in the incidence of invasive disease caused by certain serotypes, so-called secular trends, had been described for decades and this was the main hypothesis competing against serotype replacement in the early years after introduction of PCV7 (25).

Several antimicrobial drug-resistant strains were serotypes ultimately included in PCV7. Therefore, reductions in antimicrobial drug-resistant IPD were much anticipated and ultimately realized early on (17) and after several years of use of PCV7 (26). However, an observation compounding fears regarding serotype replacement was that serotype 19A was the non-PCV7 serotype with the greatest increase in incidence and that it was also associated with multidrug resistance (27). These findings suggested that inappropriate antimicrobial drug use was playing a role in the observed increases in non-PCV7 serotypes. Another hypothesis to explain the increase in serotype 19A after introduction of PCV7 was that genetic recombination events, whereby a PCV7 serotype could incorporate the genetic sequences of a non-PCV7 serotype, were occurring. So-called capsular switching might have contributed to increasing non-PCV7 serotype disease (28).

Ultimately, each of these mechanisms was shown to play a role. A systematic review of surveillance data from around the world, with EIP data being the primary contributor from North America, showed that increases in nonPCV7 serotypes were quite common in many settings and with many schedules of PCV7. However, in none of those settings did the increases in non-PCV7 IPD overshadow the reductions in PCV7-type IPD in children $<5$ years of age (29). Secular trends appeared to be a minor contributor in the United States, where epidemic serotypes 1 and 5 are relatively uncommon. Antimicrobial drug use probably influenced selection of antimicrobial drug-resistant strains among those serotypes (e.g., 19A) destined to cause replacement disease $(30,31)$. Finally, capsular switching clearly occurred but played a minor role in the increases in non-PCV7 serotypes (28). In some settings, improvements in surveillance methods at or after the time of PCV7 introduction might have falsely enhanced the increase in IPD caused by nonvaccine serotypes (32).

Worries concerning serotype replacement were tempered to an extent by the anticipated licensure of PCV13 in 2010. PCV13 included the same serotypes as PCV7 plus 6 additional types: 1, 3, 5, 6A, 7F, and, most important, 19A, the dominant replacement serotype worldwide. The US Food and Drug Administration licensed PCV13, and ACIP voted to recommend its use for children in February 2010 (33). PCV13 had large and immediate effects, in part 


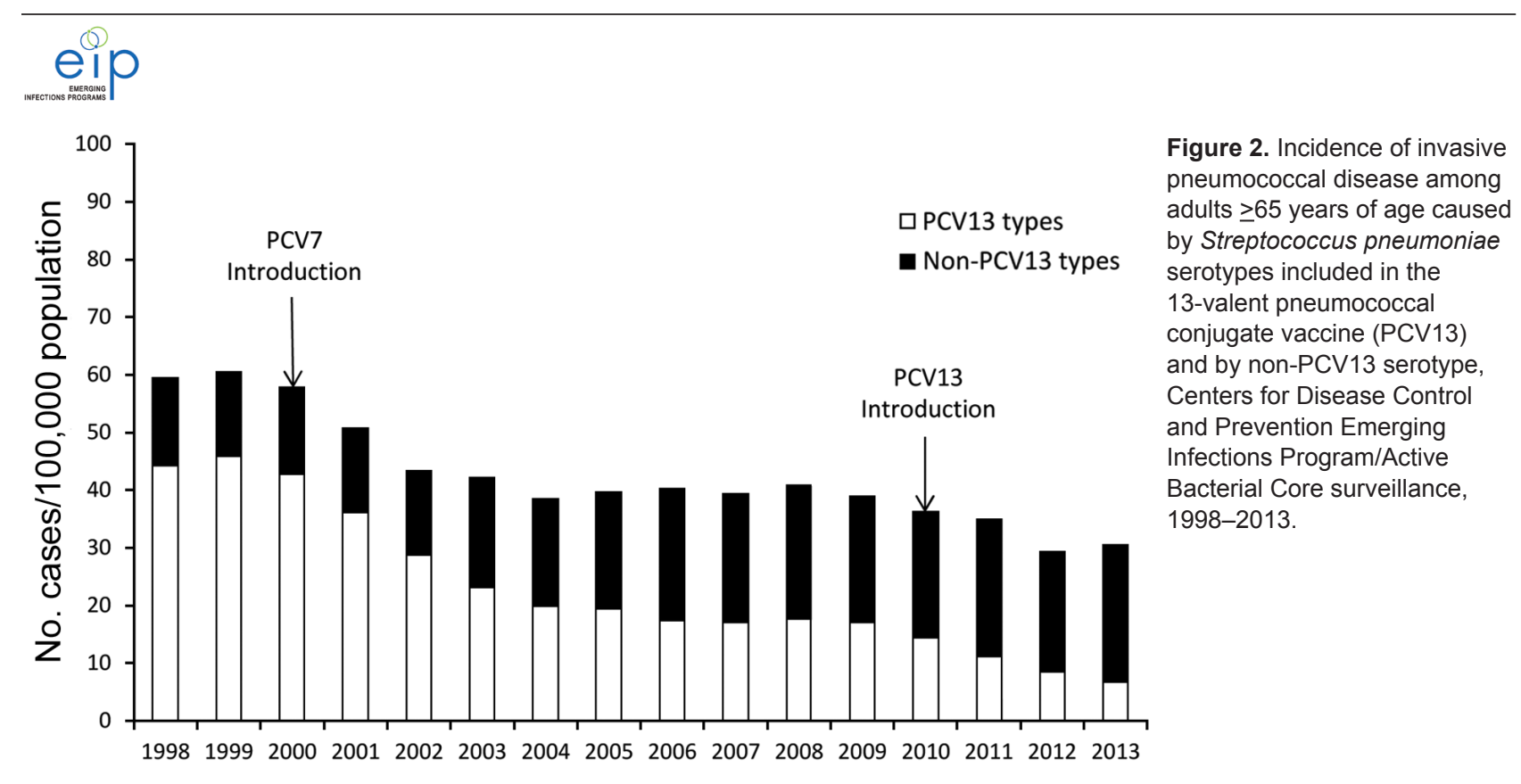

because it was licensed on the same schedule as PCV7, which enabled rapid swapping out of PCV7 for PCV13. Coverage increased rapidly, and by the end of 2011, EIPs identified reductions in PCV13-type IPD, not only among children but also among adults (34). In the short term, the benefits of PCV13 appeared comparable with those of PCV7 and have resulted in large reductions in serotypes that caused most replacement disease after widespread PCV7 use (19A and 7F). Nonetheless, more time is needed to determine whether remaining nonvaccine types will cause extensive replacement disease.

The PCV7 immunization program for children also benefited persons with immunocompromising conditions. After PCV7 introduction, rates of IPD caused by PCV7 serotypes among adults with HIV infection decreased substantially. When PCV13 was licensed for adults in 2011, ACIP discussed the possibility of recommending that vaccine for adults with immunocompromising conditions, including HIV (35). Rates of PCV7-type IPD among HIVinfected adults had remained extremely high despite having decreased from their pre-PCV7 baseline (36). Around the same time, a randomized controlled trial of PCV7 in HIVinfected adults in Malawi showed PCV7 to be $74 \%$ effective in preventing PCV7-type IPD. ACIP considered, among others, these 2 factors - high remaining burden of PCV7-type IPD among HIV-infected adults in the EIPs and demonstrated efficacy of PCV7-in ultimately recommending PCV13 for immunocompromised adults (37). On the basis of similar EIP data on disease burden among adolescent children, the ACIP ultimately recommended PCV13 for that population as well (38).

The most recent and perhaps widest-ranging change in ACIP recommendations came about in August 2014, when PCV13 was recommended for every adult $\geq 65$ years of age in the United States (39). After initially refraining from recommending PCV13 for this group (35), the ACIP reviewed extensively results of a randomized controlled trial in the Netherlands, which became available in early 2014 and showed that PCV13 was $76 \%$ effective in preventing PCV13-type IPD among persons $\geq 65$ years of age and $45 \%$ effective against non-invasive pneumonia caused by PCV13 serotypes (40). However, if there were no PCV13-type disease remaining, the ACIP might not have ever recommended the vaccine for this population of 44 million adults. Instead, data from the EIPs were instrumental in demonstrating that, despite major reductions in rates of PCV7- and PCV13-type IPD among adults, the remaining disease burden was sufficiently high that a universal, age-based recommendation was cost-effective in the short term (39).

Pneumococcal disease epidemiology has changed substantially in the United States in the past 20 years because of new prevention measures. Disease has decreased, first as a result of PCV7 introduction and, most recently, as a result of PCV13 introduction. EIPs have documented the effects of this vaccine on disease in children, disease in adults, and antimicrobial drug resistance and have provided data that helped to refine vaccine policy in the United States and elsewhere. The EIPs have elucidated the complex mechanisms at play when increases in nonvaccinetype disease are observed after reductions in vaccine-type disease and when antimicrobial drug resistance increases in response to inappropriate antimicrobial drug use and decreases in response to vaccination. In addition, the EIPs have contributed in fundamental ways to every pneumococcal vaccine recommendation in the United States since 2000. For these reasons, the EIPs have reason to celebrate their 20th anniversary. 
This study was supported by the Emerging Infections Program at the Centers for Disease Control and Prevention.

Dr. Moore is a medical epidemiologist at the Centers for Disease Control and Prevention, Atlanta, Georgia. His research interests include pneumococcal disease epidemiology, vaccinology, and prevention of pneumonia worldwide.

Dr. Whitney is the Chief of the Respiratory Diseases Branch at the Centers for Disease Control and Prevention, Atlanta, Georgia. Her research interests include pneumococcal disease epidemiology, prevention of pneumonia, and strategies for rapid introduction of pneumococcal vaccines worldwide.

\section{References}

1. World Health Organization. Estimated Hib and pneumococcal deaths for children under 5 years of age, 2008. 2013 [cited 2015 Feb 11]. http://www.who.int/immunization/monitoring _ surveillance/burden/estimates/Pneumo_hib/en/

2. Breiman RF, Butler JC, Tenover FC, Elliott JA, Facklam RR. Emergence of drug-resistant pneumococcal infections in the United States. JAMA. 1994;271:1831-5. http://dx.doi.org/10.1001/ jama.1994.03510470035031

3. Langley G, Schaffner W, Farley MM, Lynfield R, Bennett NM, Reingold R, et al. Twenty years of Active Bacterial Core surveillance. Emerg Infect Dis. 2015;21:1520-1528.

4. Bartlett JG, Breiman RF, Mandell LA, File TM Jr. Communityacquired pneumonia in adults: guidelines for management. The Infectious Diseases Society of America. Clin Infect Dis. 1998;26:811-38. http://dx.doi.org/10.1086/513953

5. Tunkel AR, Hartman BJ, Kaplan SL, Kaufman BA, Roos KL, Scheld WM, et al. Practical guidelines for the management of bacterial meningitis. Clin Infect Dis. 2004;39:1267-84. http://dx.doi.org/10.1086/425368

6. Robinson KA, Baughman W, Rothrock G, Barrett NL, Pass M, Lexau C, et al. Epidemiology of invasive Streptococcus pneumoniae infections in the United States, 1995-1998: opportunities for prevention in the conjugate vaccine era. JAMA. 2001;285:1729-35. http://dx.doi.org/10.1001/jama.285.13.1729

7. Greene CM, Kyaw MH, Ray SM, Schaffner W, Lynfield R, Barrett NL, et al. Preventability of invasive pneumococcal disease and assessment of current polysaccharide vaccine recommendations for adults: United States, 2001-2003. Clin Infect Dis. 2006;43:141-50. http://dx.doi.org/10.1086/505117

8. Bridges CB, Winquist AG, Fukuda K, Cox NJ, Singleton JA, Strikas RA, et al. Prevention and control of influenza: recommendations of the Advisory Committee on Immunization Practices (ACIP). MMWR Recomm Rep. 2000;49:1-38.

9. Black S, Shinefield H, Fireman B, Lewis E, Ray P, Hansen JR, et al. Efficacy, safety and immunogenicity of heptavalent pneumococcal conjugate vaccine in children. Northern California Kaiser Permanente Vaccine Study Center Group. Pediatr Infect Dis J. 2000;19:187-95. http://dx.doi.org/10.1097/ 00006454-200003000-00003

10. Centers for Disease Control and Prevention. Preventing pneumococcal disease among infants and young children: recommendations of the Advisory Committee on Immunization Practices (ACIP). MMWR Recomm Rep. 2000;49:1-35.

11. Committee on Infectious Diseases. Policy statement: recommendations for the prevention of pneumococcal infections, including the use of pneumococcal conjugate caccine (Prevnar), pneumococcal polysaccharide vaccine, and antibiotic prophylaxis. Pediatrics. 2000;106:362-6. http://dx.doi.org/10.1542/peds.106.2.362
12. Centers for Disease Control and Prevention. Updated recommendations on the use of pneumococcal conjugate vaccine in a setting of vaccine shortage-Advisory Committee on Immunization Practices. JAMA. 2002;287:833-4. http://dx.doi.org/ 10.1001/jama.287.7.833

13. Centers for Disease Control and Prevention. Updated recommendations on the use of pneumococcal conjugate vaccine: suspension of recommendation for third and fourth dose. MMWR Morb Mortal Wkly Rep. 2004;53:177-8.

14. Whitney CG, Pilishvili T, Farley MM, Schaffner W, Craig AS, Lynfield R, et al. Effectiveness of seven-valent pneumococcal conjugate vaccine against invasive pneumococcal disease: a matched case-control study. Lancet. 2006;368:1495-502. http://dx.doi.org/10.1016/S0140-6736(06)69637-2

15. Centers for Disease Control and Prevention. Direct and indirect effects of routine vaccination of children with 7 -valent pneumococcal conjugate vaccine on incidence of invasive pneumococcal disease - United States, 1998-2003. MMWR Morb Mortal Wkly Rep. 2005;54:893-7.

16. Bogaert D, De Groot R, Hermans PW. Streptococcus pneumoniae colonisation: the key to pneumococcal disease. Lancet Infect Dis. 2004;4:144-54. http://dx.doi.org/10.1016/S1473-3099(04)00938-7

17. Whitney CG, Farley MM, Hadler J, Harrison LH, Bennett NM, Lynfield R, et al. Decline in invasive pneumococcal disease after the introduction of protein-polysaccharide conjugate vaccine. N Engl J Med. 2003;348:1737-46. http://dx.doi.org/10.1056/ NEJMoa022823

18. Lexau CA, Lynfield R, Danila R, Pilishvili T, Facklam R, Farley MM, et al. Changing epidemiology of invasive pneumococcal disease among older adults in the era of pediatric pneumococcal conjugate vaccine. JAMA. 2005;294:2043-51. http://dx.doi.org/10.1001/jama.294.16.2043

19. Ray GT, Whitney CG, Fireman BH, Ciuryla V, Black SB. Cost-effectiveness of pneumococcal conjugate vaccine: evidence from the first 5 years of use in the United States incorporating herd effects. Pediatr Infect Dis J. 2006;25:494-501. http://dx.doi.org/ 10.1097/01.inf.0000222403.42974.8b

20. Ray GT, Pelton SI, Klugman KP, Strutton DR, Moore MR. Cost-effectiveness of pneumococcal conjugate vaccine: an update after 7 years of use in the United States. Vaccine. 2009;27:6483-94. http://dx.doi.org/10.1016/j.vaccine.2009.08.045

21. Stoecker C, Hampton LM, Link-Gelles R, Messonnier ML, Zhou F, Moore MR. Cost-effectiveness of using 2 vs 3 primary doses of 13-valent pneumococcal conjugate vaccine. Pediatrics. 2013;132:e324-32. http://dx.doi.org/10.1542/peds.2012-3350

22. Bergman A, Hjelmgren J, Ortqvist A, Wisloff T, Kristiansen IS, Hogberg LD, et al. Cost-effectiveness analysis of a universal vaccination programme with the 7-valent pneumococcal conjugate vaccine (PCV-7) in Sweden. Scand J Infect Dis. 2008;40:721-9. http://dx.doi.org/10.1080/00365540802014872

23. Rozenbaum MH, van Hoek AJ, Fleming D, Trotter CL, Miller E, Edmunds WJ. Vaccination of risk groups in England using the 13 valent pneumococcal conjugate vaccine: economic analysis. BMJ. 2012;345:e6879. http://dx.doi.org/10.1136/bmj.e6879

24. Singleton RJ, Hennessy TW, Bulkow LR, Hammitt LL, Zulz T, Hurlburt DA, et al. Invasive pneumococcal disease caused by nonvaccine serotypes among Alaska Native children with high levels of 7-valent pneumococcal conjugate vaccine coverage. JAMA. 2007;297:1784-92. http://dx.doi.org/10.1001/ jama.297.16.1784

25. Feikin DR, Klugman KP. Historical changes in pneumococcal serogroup distribution: implications for the era of pneumococcal conjugate vaccines. Clin Infect Dis. 2002;35:547-55. http://dx.doi.org/10.1086/341896

26. Hampton LM, Farley MM, Schaffner W, Thomas A, Reingold A, Harrison LH, et al. Prevention of antibiotic-nonsusceptible 
Streptococcus pneumoniae with conjugate vaccines. J Infect Dis. 2012;205:401-11. http://dx.doi.org/10.1093/infdis/jir755

27. Pai R, Moore MR, Pilishvili T, Gertz RE, Whitney CG, Beall B. Postvaccine genetic structure of Streptococcus pneumoniae serotype 19A from children in the United States. J Infect Dis. 2005;192:1988-95. http://dx.doi.org/10.1086/498043

28. Brueggemann AB, Pai R, Crook D, Beall B. Vaccine escape recombinants emerge after pneumococcal vaccination in the United States. PLoS Pathog. 2007;3:e168. http://dx.doi.org/ 10.1371/journal.ppat.0030168

29. Feikin DR, Kagucia EW, Loo JD, Link-Gelles R, Puhan MA, Cherian T, et al. Serotype-specific changes in invasive pneumococcal disease after pneumococcal conjugate vaccine introduction: a pooled analysis of multiple surveillance sites. PLoS Med. 2013;10:e1001517. http://dx.doi.org/10.1371/ journal.pmed.1001517

30. Porat N, Benisty R, Trefler R, Givon-Lavi N, Dagan R. Clonal distribution of common pneumococcal serotypes not included in the 7-valent conjugate vaccine (PCV7): marked differences between two ethnic populations in southern Israel. J Clin Microbiol. 2012;50:3472-7. http://dx.doi.org/10.1128/JCM.01309-12

31. Song JH, Dagan R, Klugman KP, Fritzell B. The relationship between pneumococcal serotypes and antibiotic resistance. Vaccine. 2012;30:2728-37. http://dx.doi.org/10.1016/j.vaccine.2012.01.091

32. Hanquet G, Valenciano M, Simondon F, Moren A. Vaccine effects and impact of vaccination programmes in post-licensure studies. Vaccine. 2013;31:5634-42. http://dx.doi.org/10.1016/ j.vaccine.2013.07.006

33. Nuorti JP, Whitney CG. Prevention of pneumococcal disease among infants and children - use of 13-valent pneumococcal conjugate vaccine and 23-valent pneumococcal polysaccharide vaccinerecommendations of the Advisory Committee on Immunization Practices (ACIP). MMWR Recomm Rep. 2010;59:1-18.

34. Moore MR, Link-Gelles R, Schaffner W, Lynfield R, Lexau C, Bennett NM, et al. Effect of use of 13-valent pneumococcal conjugate vaccine in children on invasive pneumococcal disease in children and adults in the USA: analysis of multisite, population-based surveillance. Lancet Infect Dis. 2015;15:301-9. http://dx.doi.org/10.1016/S1473-3099(14)71081-3

35. Centers for Disease Control and Prevention. Licensure of 13-valent pneumococcal conjugate vaccine for adults aged 50 years and older. MMWR Morb Mortal Wkly Rep. 2012;61:394-5.

36. Cohen AL, Harrison LH, Farley MM, Reingold AL, Hadler J, Schaffner W, et al. Prevention of invasive pneumococcal disease among HIV-infected adults in the era of childhood pneumococcal immunization. AIDS. 2010;24:2253-62. http://dx.doi.org/10.1097/ QAD.0b013e32833d46fd

37. Centers for Disease Control and Prevention. Use of 13-valent pneumococcal conjugate vaccine and 23 -valent pneumococcal polysaccharide vaccine for adults with immunocompromising conditions: recommendations of the Advisory Committee on Immunization Practices (ACIP). MMWR Morb Mortal Wkly Rep. 2012;61:816-9.

38. Centers for Disease Control and Prevention. Use of 13-valent pneumococcal conjugate vaccine and 23-valent pneumococcal polysaccharide vaccine among children aged 6-18 years with immunocompromising conditions: recommendations of the Advisory Committee on Immunization Practices (ACIP). MMWR Morb Mortal Wkly Rep. 2013;62:521-4.

39. Tomczyk S, Bennett NM, Stoecker C, Gierke R, Moore MR, Whitney CG, et al. Use of 13-valent pneumococcal conjugate vaccine and 23 -valent pneumococcal polysaccharide vaccine among adults aged $\geq 65$ years: recommendations of the Advisory Committee on Immunization Practices (ACIP). MMWR Morb Mortal Wkly Rep. 2014;63:822-5.

40. Bonten M, Bolkenbaas M, Huijts S, Webber C, Gault S, Gruber W, et al. Community-acquired pneumonia immunisation trial in adults (CAPITA) [abstract]. Pneumonia. 2014;3:95 [cited 2015 May 21]. https://pneumonia.org.au/index.php/pneumonia/pages/view/isppd

Address for correspondence: Matthew R. Moore, Centers for Disease Control and Prevention, 1600 Clifton Rd NE, Mailstop C23, Atlanta, GA 30329-4027, USA; email: zdn4@cdc.gov

\section{Table of Contents}

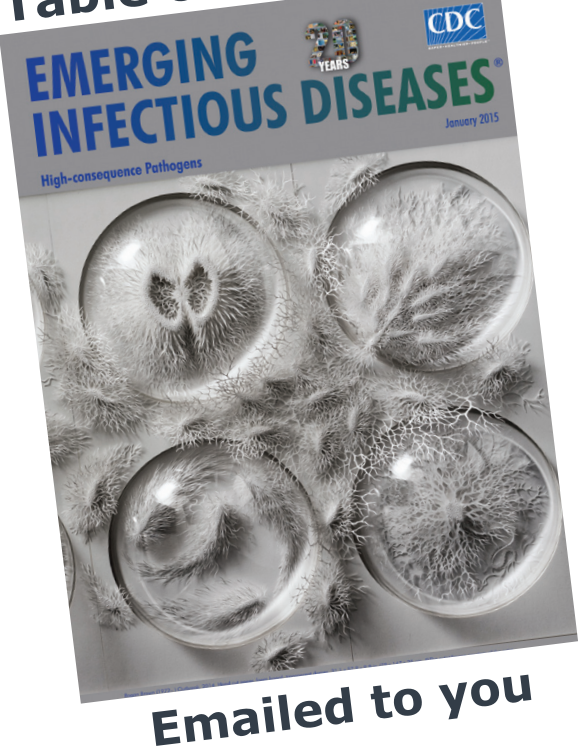

\section{GovDelivery}

\section{Manage your email alerts} so you only receive content of interest to you.

\author{
Sign up for an Online Subscription: \\ wwwnc.cdc.gov/eid/subscribe.htm
}

